\title{
A Utilização dos Serviços de Atenção Básica e de Urgência no SUS de Belo Horizonte: problema de saúde, procedimentos e escolha dos serviços'
}

\section{The utilization of Primary Care and Emergency services in the SUS of Belo Horizonte: health problems, procedures and choice of services}

\author{
Maria Raquel Gomes Maia Pires \\ Pós-doutora em arte e lúdico na educação para a saúde. Profes- \\ sora Adjunta do Departamento de Enfermagem da Universidade \\ de Brasilia (UnB). \\ Endereço: SHIN CA 05 BL-I, ap. 528, Lago Norte, CEP 71503-505, \\ Brasília, DF, Brasil. \\ E-mail: maiapœunb.br

\section{Leila Bernarda Donato Göttems} \\ Doutora em Administração, Professora da Universidade Católica \\ de Brasília e da Escola Superior de Ciências da Saúde da Secretaria \\ de Saúde do Distrito Federal. \\ Endereço: QI 25, Lote 10, ap. 214, Guará II, CEP 71060-260, Brasília, \\ DF, Brasil. \\ E-mail: leilad®ucb.br \\ Tiago Vilela Cupertino \\ Bacharel em Enfermagem. \\ Endereço: Avenida José Cândido da Silveira, 109, apto 302, Bairro \\ Cidade Nova, CEP 31170-193, Belo Horizonte, MG, Brasil. \\ E-mail: tiagocupertinoळgmail.com \\ Lucas Simões Leite \\ Bacharel e Licenciado em Enfermagem, colaborador da pesquisa. \\ Endereço: Rua Bombaim, 72 - Trevo, CEP 31370-220, Belo Horizonte, \\ MG, Brasil. \\ E-mail: lucas.s.leiteœhotmail.com

\section{Luciana Ribeiro do Vale} \\ Bacharel em Enfermagem, Trainee do Grupo Fleury: medicina e \\ saúde. \\ Endereço: Av. Dr. Luis Rocha Miranda 528, ap. 33A, CEP $04344^{-0}$-10, \\ São Paulo, SP, Brasil. \\ E-mail: luciana.valeœfleury.com.br
}

\author{
Marcelo Augusto de Castro \\ Mestrando pela Escola Enfermagem da UFMG. \\ Endereço: Rua Teixeira Leite, 107, casa 02, João Pinheiro, CEP 30530- \\ 280, Belo Horizonte, MG, Brasil. \\ E-mail: macastroenfळyahoo.com.br \\ Ana Carolina Alves Lage \\ Bacharelado e Licenciatura em Enfermagem; Gerente Comercial \\ da empresa BULDOOR no Brasil. \\ Endereço: Calle Coronel Portillo, 364, San Isidro, CEP 27, Lima/ \\ Peru. \\ E-mail: acalmoralesळhotmail.com. \\ Túlio Gomes da Silva Mauro \\ Mestre em Psicologia; consultor em estatística. \\ Endereço: SMPW, QD. 20, CJ. 03, LT 09, CS. C, Park Way, CEP 71745- \\ 003, Brasília, DF, Brasil. \\ E-mail: tuliog®uol.com.br \\ I Financiamento: Fundação de Amparo à Pesquisa do Estado de \\ Minas Gerais, Edital PPSUS 005/06. Processo no 3301/06.
}




\section{Resumo}

Avalia-se o uso das unidades básicas e de urgências do Sistema Único de Saúde (SUS) de Belo Horizonte (BH). Objetivos: identificar o principal problema de saúde que o cidadão leva à Unidade de Prontoatendimento (UPA) e à Unidade Básica de Saúde (UBS), considerando as especificidades dos níveis de atenção; caracterizar os principais procedimentos de atenção básica e de média complexidade utilizados nesses serviços, comparativamente. Pesquisa do tipo survey, com aplicação direta de questionário fechado a 997 entrevistados, distribuídos em 10 UBSs e 7 UPAs. A demanda que chega aos serviços investigados é por consultas médicas e por procedimentos de enfermagem, motivada por afecções leves, passíveis de atendimento na atenção básica. Os usuários vão à UBS por ser um serviço próximo da residência, de rápido atendimento e de fácil deslocamento, caracterizando boa oferta e capilaridade da Saúde da Família. Verificou-se duplicidade na utilização dos serviços, o que contribui para outras investigações.

Palavras-chave: Serviços de saúde; Avaliação em saúde; Atenção Primária à Saúde.

\section{Abstract}

The use of the Primary Care and Emergency Units of Sistema Único de Saúde (SUS - Brazilian National Health System) was evaluated in the city of Belo Horizonte (Southeastern Brazil). Objectives: to identify the main health problem that the citizen takes to the Emergency Unit and to the Primary Care Unit, considering the specificities of the health care levels; to characterize the main primary level and second level procedures that are used in these services, comparatively. This is a survey in which a closed questionnaire was administered to 997 interviewees from 10 Primary Care Units and 7 Emergency Units. The demand that arrives at the investigated services is for medical consultations and nursing procedures, motivated by minor affections which can be assisted in the Primary Care Unit. Users go to the Primary Care Unit because it is a service that is close to their homes and provides quick assistance, characterizing good offer and capillarity of the Family Health Program. Duplicity in services utilization was verified, which contributes to further investigations.

Keywords: Health Services; Health Evaluation; Primary Health Care. 


\section{Introdução}

Investiga-se a utilização das Unidades Básicas de Saúde (UBS) e das Unidades de Pronto-atendimento (UPA) da capital mineira pelos usuários e como se estabelecem os fluxos entre esses serviços. Têm-se como premissa que, embora o contexto e as diretrizes da política de atenção básica local influenciem o modo de produzir as ações em saúde, a utilização dos serviços pelo usuário ocorre de forma semelhante tanto em UBSs com equipes de Saúde da Família, quanto nas UPAs.

O sistema de saúde de BH está conformado em nove Distritos Sanitários, que correspondem às regiões administrativas da cidade, onde se distribuem os serviços de saúde. Aimplantação do Programa Saúde da Família (PSF) na capital mineira teve início em 2002, na tentativa de captar os profissionais da própria rede, mediante adesão voluntária estimulada por um plus salarial, e resultou na formação de 176 equipes. 0 município conta com 508 equipes de saúde da família (ESF), distribuídas em 145 Unidades Básicas de Saúde, responsáveis pela atenção básica de aproximadamente $75 \%$ da população citadina. A rede básica conta com profissionais que oferecem suporte às ações do PSF na própria UBS, como a equipe de apoio (188 médicos pediatras, 124 clínicos, 130 ginecologistas, 89 assistentes sociais), 65 equipes de saúde mental, 200 equipes de saúde bucal e 2 núcleos de reabilitação (Belo Horizonte, 2006).

Desde 1994, o Brasil elegeu a Estratégia Saúde da Família (ESF) como principal estratégia para reorganização da rede básica de serviços de saúde, apostando nas repercussões para o Sistema Único de Saúde (SUS) (Andrade, 2008). Um dos problemas que a Saúde da Família encontra, para consolidar-se enquanto mudança de práticas, consiste nas diferentes formas com que ela se implanta nos grandes centros urbanos. Em geral, devido aos problemas estruturais típicos das metrópoles (Ribeiro e col., 2006), a estratégia tende a inserir-se de forma periférica e, ou sem grandes impactos na forma de organizar as ações básicas de saúde, perpetuando o modelo tradicional de atenção. Ou seja, atenção à saúde recortada por programas de saúde pública, atendimento à demanda espontânea, fragilidade das ações de vigilância/promoção da saúde, centrados na doença e no profissional médico. Tal desvio nos princípios que regem a Estratégia Saúde da Família tem causas estruturais mais profundas, posto tratar-se de uma mudança paradigmática processual, histórica e tensa em conflitos na arena política de conformação do SUS (Göttems e Pires, 2009).

Por outro lado, se a Saúde da Família estiver inserida num processo de educação permanente, contar com condições de trabalho e rede de serviços adequada, o vínculo entre profissionais, usuário, famílias e comunidade pode melhorar consideravelmente o acesso à saúde pela população. A perspectiva do termo acesso, adotada neste artigo, contempla fatores explicativos contextuais, tais como as necessidades e a qualidade do cuidado, foca-se no domínio da efetividade das ações e na satisfação dos usuários (Travassos e Martins, 2004; Travassos e Castro, 2008; Donabedian, 1973). Neste estudo, vê-se utilização como uma dimensão do acesso, caracterizada pelo uso ou consumo do serviço de saúde ofertado no momento do atendimento (Travassos e Martins, 2004; Travassos e Castro, 2008; Travassos e col., 2006).

São objetivos da investigação: caracterizar os principais procedimentos de atenção básica e de média complexidade utilizados pelos usuários nos serviços de saúde investigados, comparativamente; identificar o principal problema de saúde que o usuário leva à Unidade de Pronto-atendimento (UPA) e à Unidade Básica de Saúde (UBS), considerando as especificidades dos níveis de atenção no SUS.

\section{Método}

Trata-se de pesquisa descritiva, do tipo survey (Babbie, 1999), com os usuários do Sistema Único de Saúde de Belo Horizonte. Inquiriu-se sobre o problema de saúde, os motivos da escolha pelo serviço e as formas de acesso às UBSs e às UPAs. As variáveis utilizadas foram: 1- problema de saúde referido: relato dos usuários sobre o problema de saúde no momento da entrevista (variável dependente); 2- tipo de atendimento realizado: procedimentos utilizados pelo usuário no momento do consumo do serviço ofertado, por nível de complexidade do SUS; 3- tempo de espera pelo serviço: tempo que o usuário levou para ser completamente atendido e para conseguir 
a consulta médica; 4- motivos da procura pela UBS e pela UPA, comparativamente; 6- serviços utilizados na UBS; 7- perfil socioeconômico do usuário: sexo, faixa etária, escolaridade, renda familiar, local de residência, local de trabalho, aquisição de plano de saúde.

Avaliaram-se os seguintes indicadores: a- comparação dos procedimentos utilizados pelos usuários nas UBSs e nas UPAs; b- problema de saúde referido pelo usuário no momento da entrevista, na UBS e na UPA; c- motivos da escolha do serviço, pelo usuário; d- serviços que o usuário utiliza na Unidade Básica de Saúde.

\section{Cenário, população e amostra do estudo}

No que se refere ao plano amostral, a população compreendeu todas as pessoas residentes no município de BH que utilizam o SUS, que corresponde a $75 \%$ segundo a Agência Nacional de Saúde (ANS). Partiu-se de uma prevalência média de $75 \%$ de consultas médicas e de procedimentos de enfermagem realizados tanto na UBS quanto na UPA, obtidos na literatura (Pires e col., 2010; Capilheira e Santos, 2006; Boing e col., 2010), independentemente do problema de saúde e nível de atenção do SUS. Com uma população de 2.000.00o de habitantes que são atendidos na rede de serviços públicos, um erro de amostragem de $3 \%$ e um nível de confiança de 95\%, estimou-se uma amostra de 820 usuários. A amostra dos serviços de saúde considerou as sete UPAs do município e dois estratos de UBS - um com unidades que possuem a população adstrita completamente coberta por equipes da Saúde da Família e outro com população apenas parcialmente coberta pela estratégia. Fez-se um sorteio aleatório, considerando a proporcionalidade de uma UBS por Distrito Sanitário, totalizando os nove da cidade. Tendo em vista que as 31 UBSs parcialmente cobertas pela ESF correspondem a $21,3 \%$ das 146 existentes no município, procurou-se manter essa proporcionalidade na amostra de serviços de atenção básica. Ao final, sortearam-se 2 UBSs parcialmente (Parcial-ESF) e 7 completamente cobertas pela Saúde da Família (UBS-ESF).

A previsão do número de usuários que seriam entrevistados em cada um dos serviços foi distribuída na proporcionalidade de $1 / 3$ da amostra total para as
UPAs e 2/3 entre as UBSs. Ao final aplicou-se o total de 993 questionários, com uma perda residual de 15 instrumentos $(0,9 \%)$, resultando em 173 questionários a mais do que a amostra calculada inicialmente, sendo $69 \%$ nas UBSs e $31 \%$ nas UPAs. No curso da pesquisa, incluiu-se mais uma UBS à amostra, totalizando 10, para garantir maior aproximação com as características epidemiológicas e sociais de um dos Distritos Sanitários.

\section{Instrumento, coleta e análise de dados}

Utilizou-se um questionário fechado para coleta de dados, adaptado de pesquisa que analisou a oferta e a demanda por média complexidade, em sua relação com atenção básica, em 7 hospitais do Distrito Federal (Pires e col, 2010). A escolha por um instrumento previamente elaborado, aplicado e testado noutra realidade justifica-se pelos seguintes motivos: a- coincidência com o objeto de estudo, ambos pautados na análise comparativa da utilização de procedimentos nos serviços de média complexidade e de atenção básica; b- rigor metodológico do processo de construção do instrumento escolhido, em que constam cinco etapas e 1 piloto (Pires e col., 2010); c- uso de variáveis e itens testados em estudos anteriores, recomendados na literatura (Sampieri, 2006; Hill e Hill, 2009). Para adaptação do referido instrumento a esta pesquisa, realizou-se um piloto, com aplicação de 103 questionários, sendo 53 numa UBS e 50 numa UPA; fizeram-se os ajustes e as modificações necessárias, adequando-os aos objetivos e ao contexto do SUS de Belo Horizonte. A versão final contempla 26 itens e 7 variáveis, suficientes para os objetivos propostos.

Optou-se pela aplicação direta dos questionários, por meio de entrevistas individuais, em que o encontro intersubjetivo com o usuário para o desvelamento das falas, devidamente registradas nos diários de campo, subsidiou a complementaridade das informações. Anotavam-se diariamente os dados sobre a quantidade de questionários previstos e realizados no dia, o fluxo de atendimento, as rotinas, a escala dos profissionais, as ocorrências e as impressões sobre os serviços de saúde que pudessem prejudicar e/ou explicar os resultados da coleta. Tais registros mostraram-se úteis no percurso e na análise dos dados. 
A entrevista foi realizada com o usuário após o atendimento, no próprio serviço de saúde, uma vez que se pretendeu aferir os procedimentos utilizados pelo cidadão. Contou-se com uma equipe de 8 entrevistadores, distribuídos nos turnos da manhã e da tarde, de segunda a sexta-feira. Estabeleceramse cotas de entrevistas por turno, dia e serviço, equilibrando-as. Em média, a coleta durou dois dias em cada um dos 17 serviços investigados e o tempo de abordagem ao entrevistado foi de 10 minutos. Os cuidados prévios e durante a coleta de dados contemplaram: a- o treinamento teórico e prático dos entrevistadores; b- a entrada no campo de maneira planejada e articulada com a Secretaria de Saúde de Belo Horizonte; c- a presença do coordenador de campo, com a função de garantir a qualidade das informações no momento da coleta, evitando perdas de questionário; d-inserção concomitante dos dados no banco, identificando possíveis erros e reparandoos com os entrevistadores quando possível; e- coleta concentrada em um único serviço, evitando pulverização das informações.

Utilizou-se a estatística descritiva básica e o pacote estatístico SPSS (Statistical Package for the Social Sciences), versão 15.0, para a análise dos aspectos conjunturais e variáveis quantitativas do banco de dados. Analisaram-se as frequências e os percentuais; fez-se teste de comparação entre frequências, qui-quadrado $x^{2}$, e de correlação de spearmans'rho para a interpretação dos resultados. 0 estudo foi aprovado pelos Comitês de Ética em pesquisa da UFMG, Parecer n ETIC 053/o6, e da Secretaria Municipal de Saúde de BH, protocolo 19/20o6.

\section{Resultados}

\section{Perfil socioeconômico e procedência}

Dos 993 usuários, 94,4\% eram residentes em BH e 5,6\% em municípios metropolitanos. Quanto ao sexo, do total, $73,2 \%$ são mulheres e $26,8 \%$ homens; a faixa etária concentrou-se entre 21 e 40 anos; $38,3 \%$ não tinham concluído o ensino fundamental, seguidos de $27,7 \%$ que concluíram o ensino médio. Aqueles usuários sem escolaridade e os que não concluíram o ensino fundamental totalizaram $44,2 \%$ dos entrevistados. Quanto ao local de trabalho, 91,5\% são empregados na capital mineira. A empregabilidade distribui-se em: empregados (33,8\%); ocupações que não possuem renda (33,2\%); inativos $(17,0 \%)$ e subempregados (16,1\%). A renda variou de um a três salários mínimos (53,8\%); muitos não têm plano de saúde $(69,6 \%)$ e quando o possuem, não passa dos $25,9 \%$.

Com exceção do local de residência e da empregabilidade, não há maiores diferenças estatisticamente significativas no perfil do usuário que utiliza os diferentes serviços públicos de saúde de $\mathrm{BH}$. Os que usam as UPAs e não moram em BH correspondem a 17,49\%; na UBS esse percentual é o,3\%. A empregabilidade dos entrevistados nas UPAs também é superior aos da atenção básica (UPA, 43,89\%; UBS, 29,23\%). Não se observou diferença significativa entre homens e mulheres quanto à escolaridade e idade, mas existe quanto à renda familiar $\left(x^{2}=72,878 ; \mathrm{gl}=5\right.$; p<,ooo); ocupação ( $x^{2}=103,422 ; g l=3$; $\left.<<, 000\right)$; local onde mora $\left(x^{2}=6,683 ; g l=1 ; p<, 010\right)$; local de trabalho $\left(x^{2}=70,838 ; g l=2 ; p<, 000\right)$ e plano de saúde $\left(x^{2}=11,289\right.$; $\mathrm{gl}=2 ; \mathrm{p}<, 004)$. Os homens demandam mais serviços de saúde na faixa etária de 11 a 30 anos $(77,8 \%)$ em relação às mulheres (55,0\%). Na faixa de idade de 31 a 40 anos, a situação inverte-se, com maior demanda por serviços sendo realizada pelas mulheres $(27,1 \%)$ em relação aos homens (10,8\%).

\section{Problema de saúde referido e utilização dos ser- viços de saúde}

O deslocamento aos serviços é feito em menos de 30 minutos para $94 \%$ dos usuários das UBSs e $77,6 \%$ daqueles que se encontravam nas UPAs, sendo estatisticamente significativo $\left(x^{2}=162,350 ; \mathrm{gl}=3\right.$; p<0,0001). As pessoas vão principalmente a pé $(85 \%)$ ou de ônibus/van/lotação/táxi (7,9\%) para as UBSs, seguidos de veículo particular (5,9\%;), moto (1\%) ou carona (o,2\%). Nas UPAs essa proporção inverte-se; utiliza-se principalmente o transporte público (43,6\%), o veículo particular $(23,4 \%)$, a caminhada (15,5\%), a ambulância/SAMU (9,2\%), a carona ou a moto $(8,3 \%)$ para se chegar ao destino. Observou-se diferença significativa entre os grupos tanto para o tempo $\left(x^{2}=39,09 ; g L=1 ; p<0,000\right)$ quanto para a forma de deslocamento $\left(x^{2}=401,712 ; g l=2 ; p<0,000\right)$.

A UBS consegue atender ao cidadão em até 2 horas, na maioria dos casos. Na UPA se consegue ser atendido nesse tempo na metade das vezes - é 
relativamente comum uma espera superior a 4 horas, o que é raro na UBS. Consegue-se mais rápido a consulta médica nas urgências, esperando meio período em $81,8 \%$ das vezes. Na UBS, essa espera é comum em 29,4\% dos casos, sendo usual esperar mais de um dia pela consulta que se foi buscar. Isso ocorre em menor frequência na UPA e a comparação entre as respostas mostrou-se estatisticamente significativa $(\mathrm{p}<\mathrm{o}, \mathrm{ooo})$.

Quando se compara a frequência média do problema de saúde referido pelos usuários nas UBSs completamente e parcialmente cobertas pela ESF com as UPAs (Tabela 1), vê-se que as afecções leves aparecem preponderantes nas urgências (57,4\%). As UBSs parcialmente cobertas pela ESF atendem proporcionalmente mais usuários com afecções leves que aquelas completamente cobertas $(\mathrm{p}<\mathrm{o}, \mathrm{ooo})$.
Ao estratificarmos esse dado (Tabela 2), vê-se que as afecções leves como problema de saúde referido variam de $18 \%$ a $38 \%$ nas UBSs e de $51,1 \%$ a $74,4 \%$ nas UPAs.

A consulta médica básica e o procedimento de enfermagem são os principais procedimentos utilizados em todos os serviços investigados, independentemente do nível de complexidade do SUS (Tabela 1). As consultas básicas aparecem em primeiro lugar, seguidas dos procedimentos de enfermagem, sem diferença estaticamente significante entre as frequências (consultas, $p<0,266$; enfermagem, $\mathrm{p}<0,711$ ).

$\mathrm{Na}$ Tabela 3, verifica-se que as UPAs 2, 4 e 7 têm como segundo procedimento mais utilizado a classificação de risco, com leve redução dos procedimentos de enfermagem. Quanto aos procedimentos

Tabela I - Número e proporção (\%) de problemas de saúde e procedimentos utilizados pelos usuários das UBSs completa ou parcialmente cobertas pela Estratégia Saúde da Família, e das UPAs de Belo Horizonte, dezembro de 2008

\begin{tabular}{|c|c|c|c|c|c|c|}
\hline \multirow{2}{*}{ Problema de Saúde } & \multicolumn{2}{|c|}{ UBS Parcial PSF } & \multicolumn{2}{|c|}{ UBS-PSF } & \multicolumn{2}{|c|}{ UPA } \\
\hline & $\mathrm{N}$ & $\%$ & $\mathrm{~N}$ & $\%$ & $\mathrm{~N}$ & $\%$ \\
\hline Consultas de retorno & 50 & 40,3 & 134 & 33,5 & 1 & 0,3 \\
\hline Afecções leves* & 34 & 27,4 & 170 & 30,9 & 174 & 57,4 \\
\hline Ações programáticas/ Controle de agravos** & 28 & 22,6 & 157 & 28,5 & 1 & 0,3 \\
\hline Afecções moderadas a graves*** & 8 & 6,5 & 19 & 3,5 & 66 & 21,8 \\
\hline Acidente/ lesão/ gravidez alto risco & 3 & 2,4 & 12 & 2,2 & 54 & 17,8 \\
\hline Outros & 1 & 0,8 & 8 & 1,5 & 7 & 2,3 \\
\hline \multirow{2}{*}{ Procedimentos Realizados } & \multicolumn{2}{|c|}{ UBS Parcial PSF } & \multicolumn{2}{|c|}{ UBS-PSF } & \multicolumn{2}{|c|}{ UPA } \\
\hline & $\mathrm{N}$ & $\%$ & $\mathrm{~N}$ & $\%$ & $\mathrm{~N}$ & $\%$ \\
\hline Consulta médica & 82 & 66,1 & 341 & 62 & 204 & 67,3 \\
\hline Classificação de risco/ Acolhimento & 22 & 17,7 & 123 & 22,4 & 124 & 40,9 \\
\hline Procedimentos de enfermagem & 84 & 27,4 & 159 & 28,9 & 94 & 31 \\
\hline Consultas de enfermagem & 3 & 2,4 & 33 & 6 & 21 & 6,9 \\
\hline Consulta odontológica/ Procedimentos odontológicos & 9 & 7,3 & 20 & 3,6 & 3 & 1 \\
\hline
\end{tabular}

*Afecções leves: gripes, amigdalites, dermatite, bronquites leves, tosse, febre, conjuntivite, dor lombar, vômito, diarréia, dor de estômago, dor no corpo, dor de dente, distúrbio nutricional, DST;

${ }^{* *}$ Ações programáticas/controle de agravos: vacinação, pré-natal, puericultura, ação educativa, tuberculose, hanseníase, diabetes, hipertensão, doença de chagas, prevenção de câncer, hipertensão, outras

***Afecções moderadas a graves: crise asmática, pico hipertensivo, cardiopatias, infecção urinária, cólica renal, sintomatologia do infarto, abcesso dentário, DPOC, tumor/câncer 
Tabela 2 - Proporção de problemas de saúde referidos pelos usuários das UBSs, Belo Horizonte, dezembro de 2008

\begin{tabular}{|c|c|c|c|c|c|c|}
\hline $\begin{array}{l}\text { Serviços de } \\
\text { saúde (\%) }\end{array}$ & $\begin{array}{l}\text { Consulta de } \\
\text { retorno (\%) }\end{array}$ & 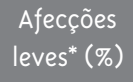 & $\begin{array}{c}\text { Ações progmáticas/Controle } \\
\text { de agravos** }(\%)\end{array}$ & $\begin{array}{c}\text { Afecções moderadas } \\
\text { a graves }{ }^{* * *}(\%)\end{array}$ & $\begin{array}{l}\text { Acidente/ lesão/ } \\
\text { gravidez alto risco (\%) }\end{array}$ & Outros (\%) \\
\hline UBS I & 26,4 & 31,2 & 39,1 & 1,1 & 1,1 & 1,1 \\
\hline UBS 2 & 26,7 & 38,3 & 26,7 & 1,6 & 6,7 & . \\
\hline UBS 3 & 58,7 & 22,2 & 17,5 & 1,6 & . & . \\
\hline UBS 4 & 25,4 & 36,5 & 25,4 & 9,5 & 3,2 & . \\
\hline UBS 5 & 35 & 26,7 & 36,7 & ।,7 & . & . \\
\hline UBS 6 & 20,8 & 34,1 & 30,76 & 6,59 & 2,19 & 5,49 \\
\hline UBS 7 & 32,8 & 37,5 & 23,4 & 4,7 & 1,6 & . \\
\hline UBS 8 & 37,1 & 30,6 & 24,2 & $\mathrm{I}, 7$ & 3,2 & 3,2 \\
\hline UBS 9 & 55,7 & 18 & 19,8 & 3,3 & 1,6 & 1,6 \\
\hline UBS 10 & 38,1 & 25,4 & 25,4 & 7,9 & 3,2 & . \\
\hline UPA I & . & 51,1 & 15,6 & 28,9 & 4,4 & . \\
\hline UPA 2 & . & 54,8 & 28,6 & 11,9 & 4,7 & . \\
\hline UPA 3 & . & 44,2 & . & 18,6 & 32,6 & 4,6 \\
\hline UPA 4 & . & 69,8 & . & 20,9 & 9,3 & . \\
\hline UPA 5 & . & 51,1 & 15,6 & 28,9 & 4,4 & . \\
\hline UPA 6 & 2,3 & 39,5 & 2,3 & 32,7 & 20,9 & 2,3 \\
\hline UPA 7 & . & 74,4 & . & 14 & II, 6 & . \\
\hline
\end{tabular}

*Afecções leves: gripes, amigdalites, dermatite, bronquites leves, tosse, febre, conjuntivite, dor lombar, vômito, diarréia, dor de estômago, dor no corpo, dor de dente, distúrbio nutricional, DST;

**Ações programáticas/controle de agravos: vacinação, pré-natal, puericultura, ação educativa, tuberculose, hanseníase, diabetes, hipertensão, doença de chagas, prevenção de câncer, hipertensão, outras

***Afecções moderadas a graves: crise asmática, pico hipertensivo, cardiopatias, infecção urinária, cólica renal, sintomatologia do infarto, abcesso dentário, DPOC, tumor/câncer

Tabela 3 - Proporção (\%) de procedimentos utilizados pelos usuários nas UBSs e UPAs de Belo Horizonte, dezembro de 2008

\begin{tabular}{|c|c|c|c|c|c|}
\hline \multirow[b]{2}{*}{ UBS } & \multicolumn{5}{|c|}{ Uso dos procedimentos informados pelos usuários } \\
\hline & $\begin{array}{l}\text { Consulta } \\
\text { médica (\%) }\end{array}$ & $\begin{array}{l}\text { Procedimentos/ } \\
\text { enfermagem (\%) }\end{array}$ & $\begin{array}{c}\text { Acolhimento/Classificação } \\
\text { de risco (\%) }\end{array}$ & $\begin{array}{l}\text { Consultas de } \\
\text { enfermagem (\%) }\end{array}$ & $\begin{array}{l}\text { Consulta procedimentos } \\
\text { odontológicos/Atendimentos } \\
\text { de outro nível superior (\%) }\end{array}$ \\
\hline UBS I & 47 & 33 & 12 & 4 & 3 \\
\hline UBS 2 & 44,44 & 24,69 & 19,75 & $\mathrm{II}, \mathrm{II}$ & . \\
\hline UBS 3 & 59,15 & 21,13 & 9,86 & 3,7 & 5,63 \\
\hline UBS $_{4}$ & 45,76 & 24,58 & 19,49 & 6,78 & . \\
\hline UBS 5 & 48,72 & 21,79 & 20,51 & . & 3,85 \\
\hline UBS 6 & 45,76 & 24,58 & 19,49 & 6,78 & . \\
\hline UBS 7 & 61 & 16,9 & 16,9 & 1,3 & 3,9 \\
\hline UBS 8 & 43,21 & 24,69 & 22,22 & 7,41 & 2,47 \\
\hline UBS 9 & 50 & 27,8 & 9,7 & . & 11,1 \\
\hline UBS 10 & 52,5 & 15 & 22,5 & 2,5 & 6,25 \\
\hline UPA I & 37,5 & 23,6 & 34,7 & 2,8 & 1,4 \\
\hline UPA 2 & 41,4 & 14,3 & 38,6 & 2,9 & 2.9 \\
\hline UPA 3 & 39,7 & 30,9 & 19,1 & 7,4 & 2,9 \\
\hline UPA 4 & 55,9 & 16,9 & 20,3 & 6,8 & . \\
\hline UPA 5 & 37,5 & 23,6 & 34,7 & 2,8 & 1,4 \\
\hline UPA 6 & 57,8 & 33,3 & 2,2 & 6,7 & . \\
\hline UPA 7 & 38,6 & 15,7 & 40 & 4,3 & 1.4 \\
\hline
\end{tabular}


de média complexidade utilizados pelos usuários da UPA, aqueles destinados à diagnose-terapia, como diagnóstico por imagem (37,04\%) e os exames de patologia clínica (30,2\%), são preponderantes, seguidos da consulta com especialistas.

O teste estatístico de correlação de Spearmans'rho entre as variáveis "problema de saúde" e "local de atendimento" teve significância elevada. Existe uma correlação importante entre a gravidade do problema de saúde referido pelo usuário e o local em que ele foi atendido $(\mathrm{r}=0,27 \mathrm{p}<0,001)$.

\section{Escolha dos serviços de saúde}

A UBS é a primeira opção de atendimento para a grande maioria usuária dos serviços básicos de saúde e para cerca da metade dos que utilizam as urgências (UBS, 9o,2\%; UPA, 52,8\%; p<0,ooo). Quanto ao motivo da procura pelo serviço (Tabela 4), predomina a proximidade da residência, o fato de ser cadastrado no local, ou por indicação de outro serviço, no caso das UPAs, com diferença significativa ( $\mathrm{p}<\mathrm{o}, \mathrm{ooo})$. Em geral, o usuário costuma procurar as emergências quando considera que o problema de saúde é grave ou quando avalia que a atenção básica não irá resolver seu problema de saúde. Vê-se na Tabela 5 que as situações de ida às UBSs são principalmente pelas consultas médicas e pelos exames de rotina. Observa-se ainda que as ações programáticas em saúde são utilizadas em maior percentual pelos usuários das UBSs parcialmente cobertas pela ESF, se comparado com aqueles das UBSs totalmente cobertas pela estratégia. Esse é o caso do acompanhamento ao crescimento e desenvolvimento infantil e da realização do pré-natal. Ocorre maior utilização das atividades educativas pelos usuários das UBS-ESF, em relação às unidades parcialmente cobertas.

Tabela ${ }_{4}$ - Número e proporção (\%) de motivos da procura pela UBS ou pela UPA, referido pelo usuário, Belo Horizonte, dezembro de 2008

\begin{tabular}{|c|c|c|c|c|c|c|}
\hline \multirow{2}{*}{ Motivos da procura } & \multicolumn{2}{|c|}{ UBS Parcial ESF } & \multicolumn{2}{|c|}{ UBS-ESF } & \multicolumn{2}{|c|}{ UPA } \\
\hline & $\mathrm{N}$ & $\%$ & $\mathrm{~N}$ & $\%$ & $\mathrm{~N}$ & $\%$ \\
\hline É mais próximo da minha residência & 80 & 64,5 & 354 & 64,4 & 134 & 44,2 \\
\hline É cadastrado nesse serviço & 17 & 3,7 & 85 & 15,5 & 1 & 0,3 \\
\hline É o único existente & 14 & 11,3 & 52 & 9,5 & 3 & 1 \\
\hline O serviço é melhor que o existente próximo à minha residência & II & 8,9 & 36 & 6,5 & 28 & 9,2 \\
\hline Foi encaminhado formalmente do CS / UPA & 0 & 0 & 7 & 1,3 & 12 & 4 \\
\hline Foi indicação / solicitação de outros serviços de saúde & 0 & 0 & 6 & 1,1 & 37 & 12,2 \\
\hline Por decisão própria, da família ou de terceiros & 0 & 0 & 4 & 0,7 & 11 & 3,6 \\
\hline Atendimento mais rápido & 0 & 0 & 1 & 0,2 & 21 & 6,9 \\
\hline Porque o CS não resolve o problema de saúde & 0 & 0 & 0 & 0 & 28 & 9,2 \\
\hline
\end{tabular}


Tabela 5 - Número e proporção (\%) de usuários segundo situações de procura a UPA e UBS, por locais de coleta, Belo Horizonte, dezembro de 2008

\begin{tabular}{|c|c|c|c|c|c|c|}
\hline \multirow[t]{2}{*}{ Situações em que procura as urgências } & \multicolumn{2}{|c|}{ UBS Parcial } & \multicolumn{2}{|c|}{ Completamente } & \multicolumn{2}{|c|}{ UPA } \\
\hline & $\mathrm{N}$ & $\%$ & N & $\%$ & $\mathrm{~N}$ & $\%$ \\
\hline Quando o problema de saúde é grave & 44 & 35,5 & 208 & 37,8 & 140 & 46,2 \\
\hline Quando fica doente e o CS não está aberto & 10 & 8,1 & 69 & 12,5 & 10 & 3,3 \\
\hline Quando avalia que o CS não vai resolver o problema & 14 & 11,3 & 61 & 11,1 & 54 & 17,8 \\
\hline $\begin{array}{l}\text { Para qualquer situação de doença, porque tem mais } \\
\text { equipamentos e já faz os exames }\end{array}$ & 1 & 0,8 & $" 1$ & 2 & 18 & 5,9 \\
\hline Para qualquer problema de saúde & 4 & 3,2 & 7 & 1,3 & 41 & 13,5 \\
\hline Pois confia no desempenho técnico dos profissionais & 0 & 0 & 3 & 0,5 & 4 & 1,3 \\
\hline \multirow[t]{2}{*}{ Situações em que procura a UBS } & \multicolumn{2}{|c|}{ UBS Parcial } & \multicolumn{2}{|c|}{ UBS } & \multicolumn{2}{|c|}{ UPA } \\
\hline & $\mathrm{N}$ & $\%$ & N & $\%$ & $\mathrm{~N}$ & $\%$ \\
\hline Consultas e exames de rotina & 112 & 90,3 & 463 & 84,2 & 153 & 50,5 \\
\hline Para pegar medicação prescrita & 90 & 72,6 & 334 & 68 & 105 & 34,7 \\
\hline Vacinação & 76 & 61,3 & 349 & 63,5 & 93 & 30,7 \\
\hline Realizar preventivo do CA ginecológico & 22 & 17,7 & 122 & 22,2 & 26 & 8,6 \\
\hline Acompanhamento de hipertensão e diabetes & 24 & 19,4 & 100 & 18,2 & 28 & 9,2 \\
\hline Solicitar encaminhamento para outro serviço e, ou especialidade & 19 & 15,3 & 100 & 18,2 & 27 & 8,9 \\
\hline Para tratamento odontológico & 26 & 21 & 53 & 9,6 & 14 & 4,6 \\
\hline Acompanhamento do CD das crianças & 15 & 12,1 & 47 & 8,5 & 24 & 7,9 \\
\hline Participar de atividades educativas & 6 & 4,8 & 43 & 7,8 & $" 1$ & 3,6 \\
\hline Para acompanhamento pré-natal & 13 & 10,5 & 32 & ।,8 & 4 & 1,3 \\
\hline Reuniões do conselho de saúde local & 1 & 0,8 & 3 & 0,5 & 1 & 0,3 \\
\hline
\end{tabular}

\section{Discussão}

Há de se destacar as características sociais da clientela atendida pelo SUS que sugere a frágil capacidade de pressão por melhores políticas públicas, tendo em vista a baixa escolaridade, renda e acesso à informação, importantes para o exercício da cidadania (Demo, 2005). Além disso, a presença maciça das mulheres na utilização dos serviços de saúde corrobora outros estudos (Ribeiro e col., 2006), que a colocam como importante protagonista no cuidado à saúde.

A procura da população pelos serviços investigados no SUS-BH é motivada por afecções leves que, na maioria dos casos, podem ser resolvidas na UBS. A duplicidade de ações entre a atenção básica e os pronto-atendimentos indica que a oferta da rede básica de BH - apesar de suficiente em termos de cobertura populacional, de estratégia organizativa e do acesso geográfico - pouco influenciou na utilização dos procedimentos por níveis de atenção. É fato curioso a indefinição de qualquer padrão de utilização de serviços nas Unidades completamente cobertas pela ESF, em relação àquelas parcialmente cobertas, o que relativiza a contribuição da Saúde da Família sobre a organização da atenção básica, no SUS Belo Horizonte.

$O$ fato da UPA figurar como a principal porta de entrada para o usuário é uma realidade usual nos serviços de saúde - seja em experiências nacionais, seja internacionais (Oliveira e Scochi, 2002; Jacobs e Matos, 2005; Mendes, 2009) - haja vista os estudos que avaliam as experiências de preservação da escolha do usuário como princípio para a reorganização dos serviços, como na Inglaterra (Green e col., 2007; Mendes, 2009). Mantidas as devidas especificidades entre os países, cabe refletir sobre como os serviços de saúde escutam e encaminham os problemas de 
saúde que lhe batem à porta, considerando a influência do modelo biomédico, ainda hegemônico nas práticas de saúde, para a reorganização das redes de cuidado no SUS (Franco e Junior, 2004; Cunha, 2005; Campos, 2007).

Porém, o fato de os usuários irem à UBS por ser o serviço mais próximo da residência, mais rápido e de fácil deslocamento indica boas perspectivas para investimentos nas mudanças do processo de trabalho dos profissionais e na rede de serviços no SUS, com impacto sobre a ampliação do acesso à saúde. Para tanto, é necessário o aperfeiçoamento das relações entre os níveis de atenção do SUS, reduzindo a duplicidade de ações para a busca da equidade nos serviços. No acesso equitativo à saúde, em lugar de se criarem demandas a partir da oferta indiscriminada de serviços, esses é que precisam estruturar-se a partir das exigências e dos problemas de saúde dos cidadãos. Há de se considerar a complexidade que a equidade agrega às políticas públicas de saúde; ou seja, no tratamento igual para necessidades iguais, na igualdade de oportunidade na utilização de serviços, no uso igualitário e a partir das necessidades humanas, ou na saúde como bem público para todos (Porto, 1995; Travassos, 1997).

A grande capilaridade que a ESF atinge associada aos desafios gerados pelo rápido crescimento quantitativo, talvez seja a maior qualidade e a maior fragilidade dessa proposta. É aspecto favorável que a implantação da Saúde da Família seja um fato no SUS difícil de ser ignorado, levantando importantes debates sobre a mudança do modelo técnico-assistencial. A Estratégia Saúde da Família, entretanto, pode retroceder quando reforma ranços antigos da saúde pública brasileira, qual seja a tendência em se constituir em ação compensatória de baixo custo para grupos marginalizados do acesso aos serviços de saúde (Campos, 2007).

O potencial de mudança da ESF reside tanto nas diretrizes que norteiam o processo de trabalho dos profissionais - integralidade, resolubilidade e intersetorialidade das ações, trabalho em equipe, vínculo de corresponsabilidade das famílias assistidas e estímulo à participação social - quanto na reorganização de práticas que ele pode ensejar (Campos, 2006; Carvalho e Cunha, 2008); isso porque, ao delimitar o território como lócus de atuação da ESF (Ministério da Saúde, 2006; Teixeira, 2002; Monken e Barcellos,
2005), pode organizar os serviços de saúde a partir da priorização dos problemas, estruturando a demanda aos demais pontos da atenção.

\section{Conclusão}

Não resolver a demanda do usuário, o atendimento lento e o acesso a consultas médicas para o mesmo dia nos serviços de atenção básica são desafios identificados no SUS de Belo Horizonte que se precisa enfrentar, dentro da proposta de reorganização dos serviços a partir da Estratégia Saúde da Família.

Há que se considerar as limitações desse estudo, que optou pela descrição dos resultados, num retrato bruto da realidade estudada, em detrimento das inferências estatísticas que se poderiam estabelecer diante dos achados. Em que pese isso, verificaram-se contradições sobre a utilização dos serviços entre os níveis de atenção do SUS, o que contribui para outras investigações. Vale questionar por que, por exemplo, sendo a Saúde da Família considerada prioritária na reorganização dos serviços, ocorre baixa utilização de ações programáticas clássicas nas UBSs em que ele foi implantado completamente. $\mathrm{Ou}$, ainda, averiguar em que medida os profissionais da atenção básica incorporaram nas suas práticas as diretrizes da estratégia saúde da família para realizarem de forma diferente as ações de promoção à saúde, de prevenção e de tratamento das doenças e quais os impactos disso sobre a utilização da rede de serviços no SUS.

\section{Referências}

ANDRADE, L. O. M.; BARRETO I. C. H. C.; BEZERRA R. C. Uma história da atenção primária à saúde. In: CAMPOS, G. W. S. et al. Tratado de saúde coletiva. São Paulo/Rio de Janeiro: Hucitec/ Fiocruz, 2008. p. 783-836.

BABBIE E. Métodos de pesquisas de survey. Belo Horizonte: Editora UFMG, 1999.

BELO HORIZONTE. Secretaria Municipal de Saúde. Relatório de Gestão 2005. Belo Horizonte: SMSA/SUS-BH, jun. 2006. Disponível em: <http://www.pbh.gov.br/smsa/biblioteca/gpld/ relatoriogesta02005.pdf >. Acesso em: o1 nov. 2008. 
BOING, A.F. Prevalência de consultas médicas e fatores associados: um estudo de base populacional no Sul do Brasil. Revista da Associação Médica Brasileira, Brasília, v. 56, n. 1, p. 41-46, 2010.

MINISTÉRIO DA SAÚDE. Gabinete do Ministro. Portaria n. 648, de março 2006. Aprova a Política Nacional de Atenção Básica, estabelecendo a revisão de diretrizes e normas para a organização da Atenção Básica para o Programa Saúde da Família (PSF) e o Programa Agentes Comunitários de Saúde (PACS). Disponível em: <http:// bvsms.saude.gov.br/bvs/saudelegis/gm/2006/ prto648_28_03_20o6.html>. Acesso em: 23 ago. 2012.

CAPILHEIRA, M. F.; SANTOS, I. S. Fatores individuais associados à utilização de consultas médicas por adultos. Revista de Saúde Pública, São Paulo, v. 4o, n. 3, p. 436-443, jun. 2006.

CAMPOS, G. W. S. O SUS entre a tradição dos sistemas nacionais e o modo liberal-privado para organizar o cuidado à saúde. Ciência \& Saúde Coletiva, Rio de Janeiro, v. 12, p. 1865-1874, 2007. Suplemento.

CAMPOS, G. W. S. Clínica e saúde coletiva compartilhadas: teoria Paideia e reformulação ampliada do trabalho em saúde. In: CAMPOS, G. W. S. et al. Tratado de saúde coletiva. São Paulo: Hucitec; Rio de Janeiro: Fiocruz, 2006. p. 53-92.

CARVALHO, S. R.; CUNHA G. T. A gestão da atenção na saúde: elementos para se pensar a mudança da organização na saúde. In: CAMPOS, G. W. S. Tratado de saúde coletiva. São Paulo: Hucitec; Rio de Janeiro: Fiocruz, 20o8. p. 837-868.

CUNHA, T. G. A construção da clínica ampliada na atenção básica. São Paulo: Hucitec, 2005.

DEMO, P. Dureza: pobreza política de mulheres pobres. Campinas: Autores Associados, 2005.

DONABEDIAN, A. Aspects of medical care administration. Cambridge: Haward University Press, 1973.
FRANCO, T. B.; JUNIOR, H. M. M. Integralidade na assistência à saúde: a organização das linhas do cuidado. In: MERHY E. E. et al. $O$ trabalho em saúde: olhando e experienciando o SUS no cotidiano. São Paulo: Hucitec, 2004.

GÖTTEMS, L. B. D; PIRES, M. R. G. M. Para além da atenção básica: reorganização do SUS por meio da interseção do setor político com o econômico. Saúde e Sociedade, São Paulo, v. 18, n. 2, 2009.

GREEN, A.; ROSS, D.; MIRZOEV, T. Primary health care and England: the coming of age of Alma Ata? Health Policy, Leeds, UK, v. 8o, p. 11-31, 2007.

HILL, M. M.; HILL, A. Investigação por questionários. Lisboa: Edições Sílabo, 2009.

JACOBS, P. C.; MATOS, E. P. Estudo exploratório dos atendimentos em unidade de emergência em Salvador-BA. Revista da Associação Médica Brasileira, São Paulo, v. 51, n. 6, p. 348-353, 2005.

MONKEN, M.; BARCELLOS, C. Vigilância em saúde e território utilizado: possibilidades teóricas e metodológicas. Cadernos de Saúde Pública, Rio de Janeiro, v. 21, n. 3, p. 898-906, 2005.

MENDES, E.V. As redes de atenção à saúde. Belo Horizonte: ESP-MG, 2009.

OLIVEIRA, M. L. F; SCOCHI, M. J. Determinantes da utilização dos serviços de urgência/emergência em Maringá-PR. Revista Ciência, Cuidado e Saúde, Maringá, v. 1, n. 1, p. 123-128, 1. sem. 2002.

PIRES, M. R. G. M et al. Oferta e demanda por média complexidade/SUS: relação com atenção básica. Ciência \& Saúde Coletiva, Rio de Janeiro, v. 15, p. 1009-1019, 2010. Suplemento 1.

PORTO, S. Justiça social, equidade e necessidade em saúde. In: PIOLA, S. F.; VIANA, S. M. Economia da saúde: conceitos e distribuição para a gestão da saúde. Brasília: IPEA, 1995. p. 123-140.

RIBEIRO, M. C. S. A et al. Perfil sociodemográfico e padrão de utilização de serviços de saúde para usuários e não-usuários do SUS - PNAD 2003. Ciência \& Saúde Coletiva, Rio de Janeiro, v. 11, n. 4, p. 1011-1022, 2006.

TEIXEIRA, C. Promoção e vigilância da saúde. Salvador: Instituto de Saúde Coletiva/UFBA, 2002. 
TRAVASSOS, C.; CASTRO, M. S. M. Determinantes e desigualdades sociais no acesso e na utilização de serviços de saúde. In: GIOVANELLA, L et al. Políticas e sistema de saúde no Brasil. Rio de Janeiro: Editora Fiocruz; 2008. p 215-46.

TRAVASSOS, C.; MARTINS, M. Uma revisão sobre os conceitos de acesso e utilização de serviços de saúde. Cadernos de Saúde Pública, Rio de Janeiro, v. 20, p. 190-8, 2004. Suplemento 2.

TRAVASSOS, C. et al. Desigualdades geográficas e sociais no acesso aos serviços de saúde no Brasil.

Revista Ciência \& Saúde Coletiva, Rio de Janeiro, v. 11, p. 975-986, 2006.

TRAVASSOS, C. Equidade e o Sistema Único de Saúde: uma contribuição para debate. Cadernos de Saúde Pública, Rio de Janeiro, v. 13, p. 325-30, 1997.

SAMPIERI, R. H. Metodologia de pesquisa. São Paulo: McGraw-Hill, 2006. 